\title{
Osteomyelitis, Osteoradionecrosis, and Medication-Related Osteonecrosis of Jaws
}

\author{
Christian Walter and Christoph Renné
}

\subsection{Introduction}

Bones belong to the connective tissue. Their main function is the internal support and source of inorganic ions. Bones have an organic matrix that is secondarily calcified with calcium salts, mainly hydroxyapatite. The organic matrix consists of a huge extent of type I collagen. Only 5\% are other proteoglycans and non-collagenous proteins [1].

There are two major bone parts: The outer compact and the interior cancellous part. In the compact bone, the collagen fibrils form concentric lamellae around a central canal that is called the Haversian canal. These canals harbor vessels which are interconnected by further vessels lying in the Volkmann's canals. The fibrils in neighboring lamellae have a perpendicular orientation resulting in higher stability of the bone. In between the calcified lamellae are therefore concentric orientated osteocytes. Their main function seems to be the mineralization of the bone [1]. The compact bone is responsible for approximately $80 \%$ of the entire bone weight [2]. The main function of the compact bone is mechanical stability whereas the cancellous bone mainly has a metabolic function [1]. The cancellous bone consists of small lamellae and has a surface 10 times bigger than the compact bone [2]. On the outside of the compact bone is the periosteum, on the inside the endosteum.

There are three kinds of bone cells: The osteoblasts, the osteocytes, and the osteoclasts.

C. Walter $(\bowtie)$

Oral and Maxillofacial Surgery - Plastic Surgery, Mediplus Clinic

Mainz, Mainz, Germany

e-mail: walter@mainz-mkg.de

C. Renné

Group Practice for Pathology Wiesbaden, Wiesbaden, Germany

\subsubsection{Osteoblasts}

Osteoblasts derive from a multipotential stem cell that differentiates via an osteoprogenitor cell into osteoblasts. Osteoblasts form new bone by the production of the inorganic matrix that mineralizes eventually. After a cycle of bone resorption and consecutive bone formation, most osteoblasts become lining cells covering the surface of the bone [1].

\subsubsection{Osteocyte}

During the course of bone formation, approximately $10 \%$ of the osteoblasts build themselves into the bony structure and become osteocytes [2]. It is assumed that the number of osteocytes is 10 times higher than the number of osteoblasts in the adult human body. The osteocytes are stellate cells that have lots of slim processes that are connected to surrounding cells. Osteocytes with their three-dimensional network seem to play the key role in bone remodeling [1].

\subsubsection{Osteoclast}

From all bone cells osteoclasts represent the smallest fraction. Osteoclasts are multinucleated giant cells that resorb bone. They derive from the monocyte macrophage line. Their only function is to resorb mineralized tissue as it is necessary for bone growth, remodeling, and tooth eruption. Most bone diseases are associated with an increased function of the osteoclasts. Therefore, osteoclasts are often the pharmaceutical target in the therapy of bone diseases such as malignancies or metabolic diseases as osteoporosis. Osteoclasts are regulated by the RANK RANKL OPG system $[1,3]$. 


\subsubsection{RANK RANKL OPG}

The receptor activator of nuclear factor $\kappa \mathrm{B}$ ligand (RANKL) is an osteoclast differentiating factor mainly expressed by osteoblasts. Secretion of RANKL leads to the differentiation of osteoclasts out of precursor cells, and the activation of osteoclasts to resorb bone by binding on RANK that is expressed on both the precursor cells and the mature osteoclasts. Osteoprotegerin (OPG) is the osteoclastogenesis inhibitor factor and functions as a decoy receptor for RANKL so that the osteoclasts cannot be activated. The RANK RANKL OPG system seems to be the most important part in the communication between the bone cells.

This system is not exclusively organizing the communication between osteoblasts and osteoclasts. This system has been proven to be responsible for the formation of the mammary gland and lymph nodes. RANK and RANKL are also expressed in the kidney, spleen, thymus, and brain, where it might influence the thermoregulation [3].

\subsection{Osteomyelitis}

The clinical picture of the different kinds of osteomyelitis is very inhomogeneous so that there are many definitions and classifications. In textbooks, the osteoradionecrosis and medication-associated osteonecrosis of the jaws are often subentities of the osteomyelitis. In this book, these entities are described in their own subheadings (Sects. 22.3 and 22.4 of the chapter).

\subsubsection{Definition}

The actual term "osteomyelitis" refers to an inflammation ("itis") of the bone marrow ("osteomyel") only but means an inflammation of the entire bone including the periosteum, the cortical, and cancellous bone as well as the bone marrow.

\subsubsection{Classification}

There are many different classifications that are either based on the etiology, pathogenesis, pathologic or anatomic differences, the clinical course of the disease, or radiologic patterns. This makes a comparison between different studies very complicated or impossible.

The Zürich classification [4] differs between three different kinds of osteomyelitis: The acute osteomyelitis, the secondary chronic osteomyelitis, and the primary chronic osteomyelitis (Table 22.1). The secondary chronic osteomyelitis results from the acute osteomyelitis and therefore is
Table 22.1 The table describes the Zürich classification of osteomyelitis on the left-hand side. In the right column are the different kinds of osteomyelitis that are included in the respective group of the Zürich classification [4]

\begin{tabular}{|c|c|}
\hline Zürich classification [4] & Different types of osteomyelitis \\
\hline \multirow{17}{*}{$\begin{array}{l}\text { Acute osteomyelitis } \\
\text { Secondary chronic } \\
\text { osteomyelitis }\end{array}$} & Neonatal osteomyelitis \\
\hline & Trauma-associated osteomyelitis \\
\hline & Odontogenic osteomyelitis \\
\hline & Foreign body-induced osteomyelitis \\
\hline & Osteomyelitis based on a bone disease \\
\hline & Osteomyelitis based on a systemic \\
\hline & - Diabetes \\
\hline & - Autoimmune diseases/ \\
\hline & $\begin{array}{l}\text { immunosuppression } \\
\text { • AIDS }\end{array}$ \\
\hline & - Agranulocytosis \\
\hline & - Anemia \\
\hline & - Leukemia \\
\hline & - Syphilis \\
\hline & - Malnutrition \\
\hline & - Cancer/chemotherapy \\
\hline & - Alcohol/tobacco/drugs \\
\hline & - Herpes zoster/Cytomegaly \\
\hline \multirow{3}{*}{$\begin{array}{l}\text { Primary chronic } \\
\text { osteomyelitis }\end{array}$} & Juvenile chronic osteomyelitis \\
\hline & Adult onset osteomyelitis \\
\hline & $\begin{array}{l}\text { Syndrome associated } \\
\text { - SAPHO } \\
\text { - CRMO }\end{array}$ \\
\hline
\end{tabular}

the same disease at a different time stage. Once the osteomyelitis persists for more than 4 weeks, it is defined as chronic [4].

\subsubsection{Epidemiology}

Due to the different classifications and terms used for the entire group of osteomyelitis, it is hard to give general data regarding its epidemiology. Approximately $17 \%$ of all osteomyelitis cases belong to the group of the acute osteomyelitis, $70 \%$ to the secondary chronic osteomyelitis, and $10 \%$ to the primary chronic osteomyelitis [4]. The average age at the time of diagnosis is a little bit over 40 years for the acute and the secondary chronic osteomyelitis [4]. Because of the inhomogeneity of the secondary chronic osteomyelitis, a general age group cannot be given.

\subsubsection{Etiology}

In the etiology of the acute osteomyelitis and the secondary chronic osteomyelitis, usually an odontogenic infection can be identified such as a dead tooth, a periodontal disease, or conditions after dentoalveolar surgery. A hematogenic spread from a different primary location into the region of the jaws is extremely rare [4]. 
The etiology of the primary chronic osteomyelitis is an infection of unknown origin [4].

\subsubsection{Pathogenesis}

The acute osteomyelitis and secondary chronic osteomyelitis are caused by a local infection due to bacteria from the oral cavity. The likelihood of the development of the infection depends on the virulence and number of bacteria and the quality of the local immune response and the blood flow [4].

Therefore, general diseases affecting the immune system are risk factors in the development of osteomyelitis, e.g., diabetes, autoimmune diseases, or anemia.

A typical course of the acute and secondary chronic osteomyelitis is the contamination of the bone with bacteria. The bacteria proliferate and colonize the bone marrow and reach via the Haversian and Volkmann canals the periosteum. The edema under and in the periosteum disturbs the blood flow resulting in ischemic bone parts and potentially sequestrum building.

\subsubsection{Histology}

The acute osteomyelitis and the secondary chronic osteomyelitis are characterized by an inflammatory exudate, primary in the medullary spaces with fibrin, leucocytes, and macrophages that replace the fatty tissue and hematopoietic marrow (Fig. 22.1). In addition, necrotic debris and bacteria can

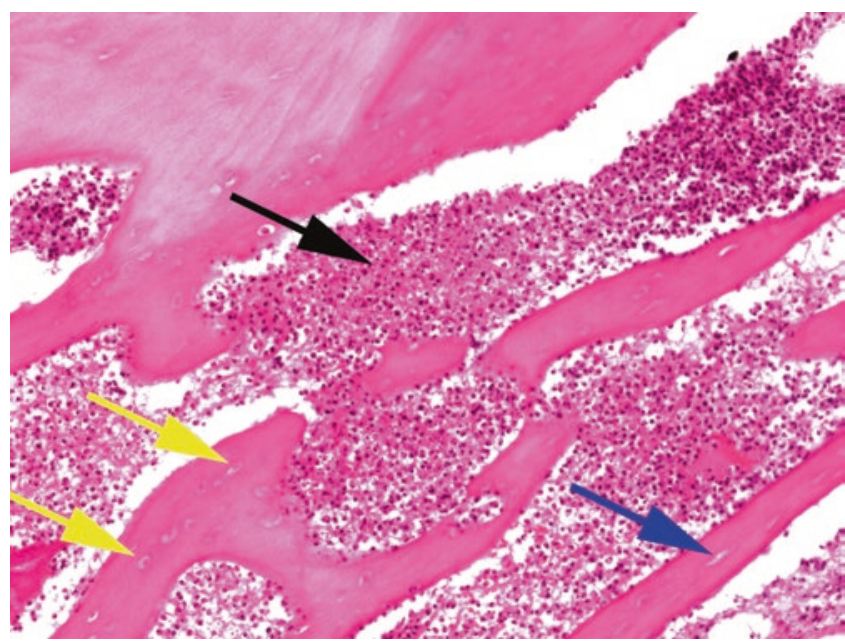

(C)Association of Oral and Maxillofacial Surgeons of India

Fig. 22.1 In this case of acute osteomyelitis, the medullary hematopoietic cells and the fatty tissue are completely replaced by cell debris, fibrin, and an inflammatory infiltrate mainly composed of neutrophil granulocytes (black arrow). Besides the vital bone with filled lacunes of osteocytes (yellow arrow), necrotic bone can be seen with loss of osteocytes (blue arrow) be found $[4,5]$. The most common bacteria being detected is Staphylococcus aureus (85\%) [4]. Due to the blood flow disturbances (see pathogenesis), parts of the bone die so that empty osteocytic lacunae can be observed. Sequestrae may be present. New bone formation under the periosteum is not uncommon.

In cases of secondary chronic osteomyelitis, sequestrum formation is more common than in the acute osteomyelitis. The more chronic the course is, the more likely is the development of bone marrow fibrosis and sclerosis of the bone. Bacteria might be present. Actinomyces drusen are typical.

In the primary chronic osteomyelitis, plasma cells are predominant in the inflammatory infiltrate. The proportion of neutrophils, lymphocytes, and macrophages is rather small. The bone marrow is altered due to fibrosis. New bone formation is a common sign. Osteoclastic activity leads to repeated bone remodeling without a distinct histological bone formation pattern. Microabscesses might be observed [4].

\subsubsection{Symptoms}

In cases of acute osteomyelitis, patients present with high fever and are listless. Local swelling can be observed with pain on palpation. The affected area is reddish, a trismus might be present, and quite often the teeth have higher mobility with pus coming out of the periodontium. If the inferior alveolar nerve is affected, patients report paresthesia of the lips (Vincent symptom [4]). There are cases in which the symptoms are not very distinctive.

Symptoms of the secondary chronic osteomyelitis are the painful swellings that are usually not as prominent as in the acute osteomyelitis. A common finding is a periosteal reaction causing a solid swelling. Further symptoms are sequester formation and fistulas.

The primary chronic osteomyelitis is characterized by a nonsuppurative inflammation and sometimes only barely noticeable symptoms. In active periods, the patients notice pain, swelling, and mouth-opening limitations. Due to the bone formation, permanent swelling will develop eventually [4].

\subsubsection{Complications}

A typical complication of the acute osteomyelitis is a shift into the chronic osteomyelitis that is very hard to treat sufficiently.

Further complications are the development of the Vincent syndrome, fistula, abscess and sequester formation, and potentially fractures [4]. 


\subsubsection{Diagnosis}

The diagnosis is based on the clinical course. This is completed by radiology: panoramic radiograph, cone beam CT, CT, or MRI. Changes in the bone can only be seen after a 30 to $40 \%$ reduction of the mineralized part of the bone. Therefore, the changes in the acute osteomyelitis are marginal at the beginning. In complex cases of osteomyelitis, a bone scintigraphy might be used to detect further active spots in the skeleton, e.g., in the diagnosis of chronic recurrent multifocal osteomyelitis or the SAPHO syndrome (SAPHO: Synovitis, acne, pustulosis, hyperostosis, osteitis) [6].

Radiological signs of acute osteomyelitis are: Bone resorption with increased radiolucency, loss of spongious structure of the bone, potentially sequester formation.

Radiological signs of the secondary chronic osteomyelitis are: Bone resorption with increased radiolucency, sequester formation, periosteal reaction, and pathological fractures.

Radiological signs of the primary chronic osteomyelitis are: Increased radiopacity with loss of trabecular bone, bone resorption, and periosteal reaction (Fig. 22.2) [4].

\subsubsection{Differential Diagnoses}

In the differential diagnosis, one should rule out malignancies in unclear cases so that biopsies should be performed.

\subsubsection{Therapy}

The therapy of the acute and secondary chronic osteomyelitis mainly consists of the therapy of the infection and of the improvement of the local blood flow. This is achieved via antibiotics and removing of the infected parts of the bone. A decortication supports this and helps to get well-vascularized tissue onto the bone.

Acute osteomyelitis is immediately treated with antibiotics. If an antibiogram suggests different antibiotics, an adaptation should be performed after the initial antimicrobiological therapy. Mouth rinses, hygiene, and cold application can be applied. In general, the highly mobile teeth should not be extracted since they will gain stability again after the acute stadium of the osteomyelitis is over.

The therapy of the secondary chronic osteomyelitis aims at sufficient pain management, limitation of the spread of the affected areas, fracture prevention, and the prevention of the onset of further active periods. Secondary diseases such as diabetes need to be treated as well.

The therapy of the primary chronic osteomyelitis consists of a surgical intervention to remove the necrotic bone parts and a potential disfigurement can be corrected. But recurrences of the symptoms are very common. Therefore, other treatment options should be used as well including antibiotics, nonsteroidal anti-inflammatory drugs (NSAIDS), steroids, and bisphosphonates (mainly pamidronate).

\subsubsection{Prognosis}

The therapeutic success is higher in patients with acute and secondary chronic osteomyelitis than in patients with primary chronic osteomyelitis. Approximately $75 \%$ of the acute and secondary chronic osteomyelitis are symptom-free after intervention whereas only about $25 \%$ are symptom-free of the patients with primary chronic osteomyelitis [4].
Fig. 22.2 Panoramic radiograph of a patient with primary chronic osteomyelitis after several surgeries have been performed. Several infusions of pamidronate prevented the occurrence of further active periods. In the left mandible, typical sclerosis can be seen with a prominent nerve canal

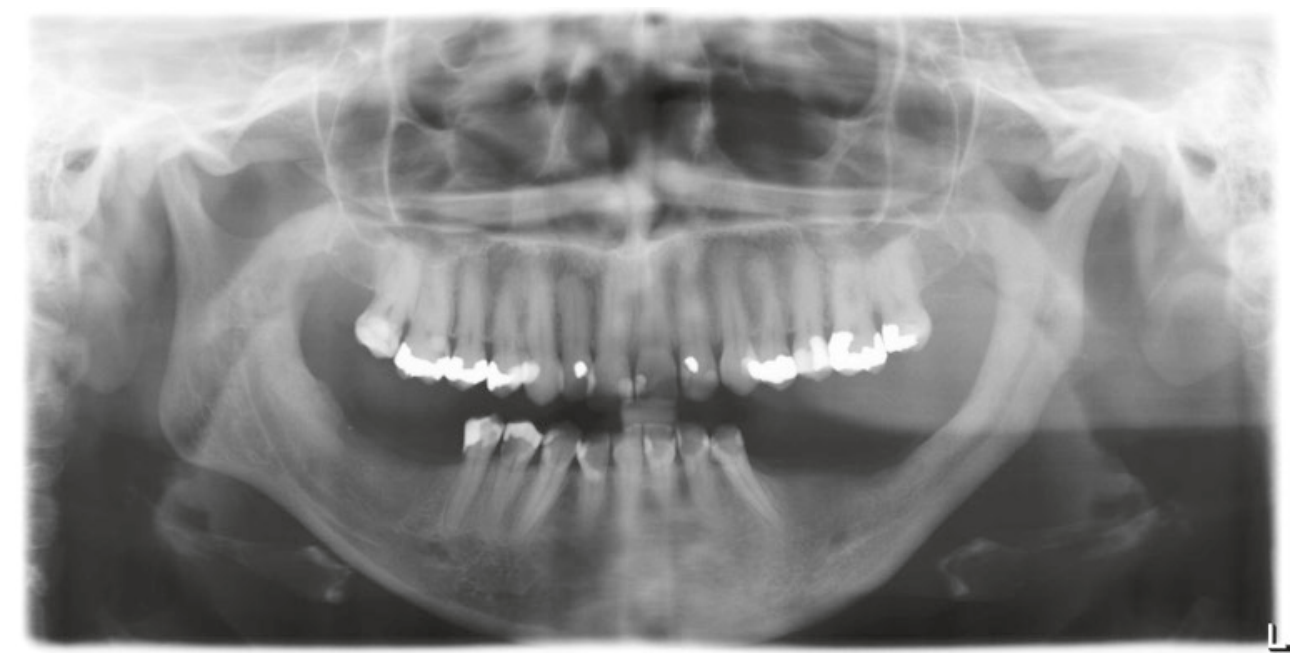

(CAssociation of Oral and Maxillofacial Surgeons of India 


\subsection{Osteoradionecrosis}

The osteoradionecrosis is a side effect in the therapy of malignant diseases to the head and neck area with ionizing radiation.

\subsubsection{Definition}

Osteoradionecrosis describes the exposed necrotic bone due to radiation. The infected osteoradionecrosis describes the additional infection of the necrotic bone [7].

\subsubsection{Epidemiology}

The prevalence of osteoradionecrosis ranges from 0 to $23 \%$ of the patients with head and neck radiation [8]. Usually, older patients are affected ( 60 years \pm 10 years) since the primary disease causing the head and neck radiation are diseases in patients with advanced age [9]. Men are affected more than twice as often [9].

\subsubsection{Etiology}

The etiology of the osteoradionecrosis is radiation therapy to the head and neck area. Usually, another trigger is required in the development of the osteoradionecrosis (see pathogenesis).

\subsubsection{Pathogenesis}

Due to the radiation therapy, the vessels of the bone change and become hyalinized. It results in a lack of nutrition and hypoxia with the subsequent death of osteocytes [7]. This seems to be more likely in radiation doses above 40-50 Gy [10]. The radiation has additional side effects to all tissues being in the radiation field including the skin, the muscles, and the salivary glands. Due to the resulting xerostomia radiation, caries develops so that an osteoradionecrosis might develop.

The osteonecrosis occurs more often in the mandible most probably to the greater extent of cortical bone and the more critical vascularization of the mandible. And the mandible most probably is more often in the radiation field due to the location of the primary tumor [10].

There are several risk factors that are associated with a more frequent occurrence of the osteoradionecrosis. Those are male gender, insufficient oral hygiene, pressure denture sores, a tumor localized in the tongue, alveolar process of the mandible, the floor of the mouth, and retromolar as well as persisting alcohol and nicotine consumption. Next to these, there are therapy-linked risk factors, namely a tumor resection including resection of the bone and dentoalveolar surgery performed in timely proximity to the radiation therapy [10].

\subsubsection{Classification}

It is mainly differed between the aseptic osteoradionecrosis and the infected osteoradionecrosis. Another very common staging system uses the potential benefit of a rather controversially discussed therapy option the hyperbaric oxygen treatment and therefore won't be discussed in further detail.

\subsubsection{Histology}

The lacunae of the osteocytes in osteoradionecrotic bone are empty (Fig. 22.3). There is a lack of osteoblastic rimming and the Haversian and Volkmann canals do not harbor any blood vessels. The marrow of the bone shows acellular collagen. The periosteum is acellular and avascular [5].

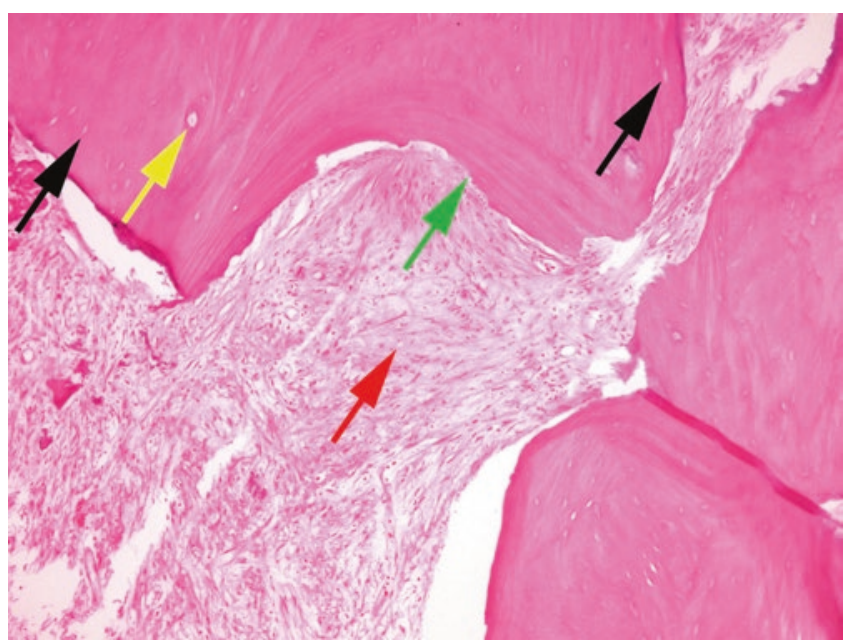

CAssociation of Oral and Maxillofacial Surgeons of India

Fig. 22.3 The examination of the osteoradionecrosis under the microscope shows avital bone with irregular fissured borders. The lacunae of the osteocytes are empty without osteocyte cell nuclei (black arrows) and also the blood vessels in the Haversian canals have undergone necrosis, so that only empty spaces are the visible remnants (yellow arrow). The necrotic bone is missing the osteoblastic rimming (green arrow). The bone marrow is replaced by a collagen fiber-rich scar like connective tissue (red arrow) 


\subsubsection{Symptoms}

Symptoms are the exposed necrotic bone that can be visible or palpable with a probe. Further symptoms are pain, swelling, the development of granulation tissue, foetor ex ore, oral and extraoral fistulas, potentially increased mobility of the adjacent teeth, altered sensibility, and occlusion [10].

\subsubsection{Complications}

Main complications are extended infections of the site, the development of fistulas, and pathological fractures. This might result in resections of the altered bone including the loss of the continuity of the bone or the opening of the nasal cavity or sinus. This results in a reduced masticatory function. Due to the fibrosis of the soft tissues, trismus might occur. All these factors lead to a reduced quality of life [10].

\subsubsection{Diagnosis}

The diagnosis is a clinical one. There needs to be a mandatory head and neck radiation and the exposed necrotic bone.

Typical radiologic findings are bone destruction, altered bone density, the occurrence of sequestra, and pathological fractures.

\subsubsection{Differential Diagnoses}

Differential diagnoses are osteomyelitis and the medicationassociated osteonecrosis of the jaws as well as the existence of malignancy so that a histological sample should be assessed.

\subsubsection{Therapy}

As already mentioned, the evidence of the use of hyperbaric oxygen treatment is rather low and is not recommended [11, 12]. Smaller necrotic areas can be treated conservatively using antiseptic mouth rinses, antibiotics, and pain killers. In some cases, a wait and see strategy is reasonable. Sometimes circumscribed debridement might be performed. Extended necrotic areas should be surgically removed [13]. The aim is to preserve damage to the alveolar nerve and to avoid a continuity defect of the mandible. The operation should be as atraumatic as possible. The periosteum should not be removed if possible. The necrotic areas should be removed, sharp edges need to be smoothened, and the bone should be covered with soft tissue. The surgery should be performed with perioperative antimicrobial therapy.

\subsubsection{Prognosis}

The risk for a recurrence of an osteoradionecrosis is high. Pressure denture sores, wound healing deficiencies, and mucositis are risk factors for a recurrence.

\subsubsection{Prevention}

A 3D-planning of radiation therapy might help to reduce the radiation doses in the bone. In addition, a splint inserted in the patient's mouth during the radiation might help to reduce the burst of the local mucosal membranes due to secondary radiation that might occur due to metallic dental restorations. An additional splint to provide fluorides to strengthen the teeth might help prevent the development of radiation caries.

It is feasible to reduce the existence of risk factors that might later on trigger the development of an osteoradionecrosis. Therefore, an initial dental checkup and therapy preceding the radiation therapy should be performed including the extraction of all non-restorable teeth. The patient should be motivated for exceptional good oral hygiene [10].

\subsection{Medication-Associated Osteonecrosis of the Jaws}

There are several pharmaceutical agents that cause osteonecrosis of the jaws. In the following, the main focus will be on the bisphosphonate-associated osteonecrosis.

\subsubsection{Bisphosphonate-Associated Osteonecrosis of the Jaws}

\subsubsection{Bisphosphonates}

Bisphosphonates are used in patients with an increased osteoclastic activity due to malignancy such as solid tumors with osseous metastases or the multiple myeloma or due to metabolic bone diseases such as osteoporosis. The main target of bisphosphonates that can be administered orally of IV is the osteoclasts.

Bisphosphonates are classified into nitrogen- and nonnitrogen-containing bisphosphonates. The non-nitrogencontaining bisphosphonates are built into ATP and can no longer be used as a source of energy in the cells. The nitrogen-containing bisphosphonates inhibit the farnesyl pyrophosphate synthase in the mevalonate pathway leading to decreased osteoclastic function [14]. In 2003, the bisphosphonate-associated osteonecrosis was first mentioned in a scientific paper [15]. 


\subsubsection{Definition}

The bisphosphonate-associated osteonecrosis is defined as the occurrence of the necrotic bone of the jaws that has been persistent for at least 8 weeks with a current or previous history of bisphosphonate use (Fig. 22.4). Furthermore, it is demanded that the patient had had no former head and neck radiation [16].

The last demand seems arbitrary since the coexistence of a further risk factor simply increases the risk of osteonecrosis development so that there is a special risk constellation. There is evidence that it is even possible to histologically distinguish an osteonecrosis derived from bisphosphonates compared to osteoradionecrosis [17].

\subsubsection{Epidemiology}

The prevalence and incidence of the bisphosphonateassociated osteonecrosis of the jaws depend on the primary disease, comedication, and the existence of local trigger factors. The highest risk is present in oncologic patients with

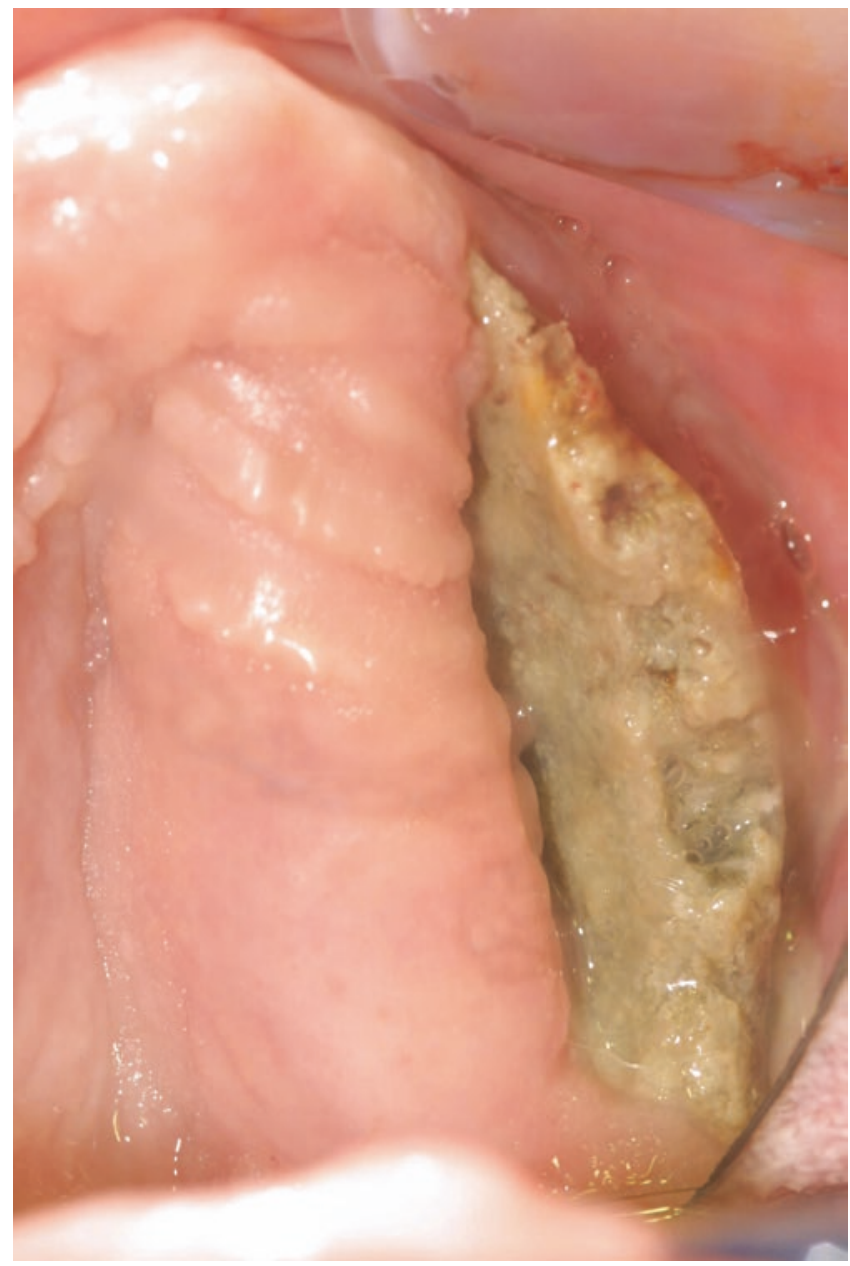

CAssociation of Oral and Maxillofacial Surgeons of India

Fig. 22.4 Huge bisphosphonate-associated osteonecrosis of the left maxilla in a patient with non-Hodgkin lymphoma and a zoledronate treatment for 2.5 years before a pressure denture sore triggered the osteonecrosis further compromising medications. Incidences for patients with malignant diseases range from 1 to $20 \%$ [18-20]. The prevalence of secondary osteoporosis is about $1 \%$ and for primary osteoporosis $0.1 \%$ [21]. There is only a small difference between the genders. Women are affected a little bit more often than men. Most probably due to the osteoporosis and breast cancer cases vs. the prostate cancer cases that exclusively occur in men. The average age is approximately 60 years \pm 10 years (standard deviation) [9].

\subsubsection{Etiology}

The main factor is the use of nitrogen-containing bisphosphonates. Administered bisphosphonates will be incorporated in the bone. It is unclear if and how long these bisphosphonates are active. The development of bisphosphonate-associated osteonecrosis can be triggered by oral factors - this is usually a wound in the oral cavity: periodontal disease, surgical procedures, etc. [9, 22].

\subsubsection{Pathogenesis}

The main target of the bisphosphonates is the osteoclast, thereby inducing reduced bone remodeling. Due to the unspecific interaction with cells, not only the osteoclasts are affected, but also, in lower concentrations, osteoblasts are stimulated [23]. The effect is an increase in total bone. Other affected cell lines are blood vessel cells, fibroblasts, and keratinocytes. The antiangiogenic function of bisphosphonates [24, 25] leads to decreased vascular exploitation [26]. This results in fewer potentially less potent vessels that have to support more bone. In addition, the soft tissues covering the bone are affected by the bisphosphonates so that potential wounds are less prone to heal [24]. In older articles, it is often described that tooth extractions are responsible for the development of the bisphosphonate-associated osteonecrosis of the jaws. This theory might not be right. It is more likely that these teeth have been extracted too late since they were extracted out of an alreadyexisting, altered, infected, or necrotic bone [27].

\subsubsection{Classification}

The bisphosphonate-associated osteonecrosis of the jaws is classified into several different stages. At-risk patients are all those receiving bisphosphonates.

Stage 0 patients are patients that do have some symptoms without any visible uncovered bone.

Stage I, the necrotic bone becomes visible. Patients in this stage usually do not have any symptoms.

Stage II, an additive infection is existent.

Stage III, further complications occur such as necrotic areas involving the base of the mandible or the sinus or pathologic fractures are existent [16]. 


\subsubsection{Histology}

Usually, there is a mucosal damage above the necrosis, perhaps some granulation tissue (Fig. 22.5). The necrotic bone is often avascular and covered in bacteria, especially Actinomyces (Fig. 22.6) [28]. The osteonecrotic bone has

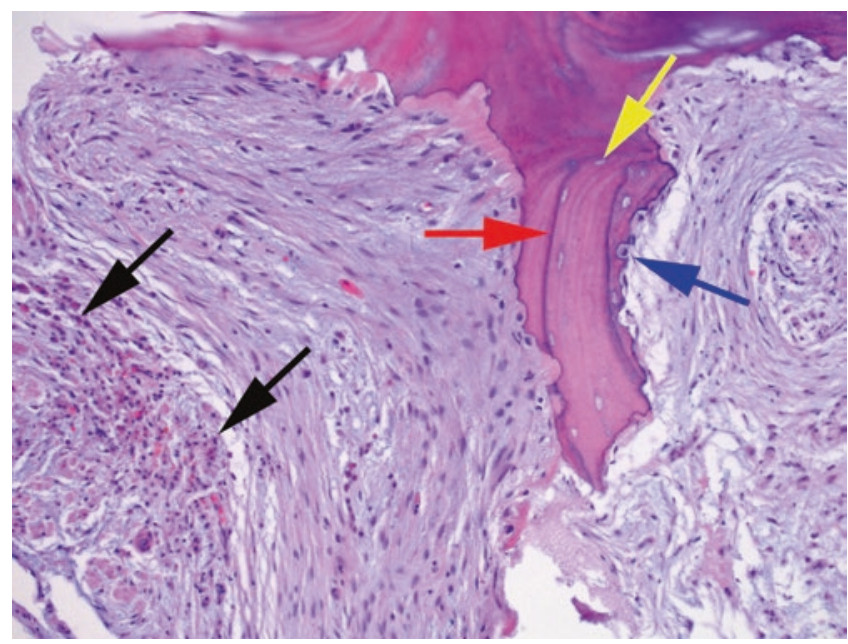

CAssociation of Oral and Maxillofacial Surgeons of India

Fig. 22.5 In this case, the bone necrosis is induced by bisphosphonates. Besides necrotic bone showing empty osteocyte lacunes (yellow arrow), inflammatory cellular infiltrate can be seen in the medullary cavity (black arrows). It is mainly composed of lymphocytes, neutrophil granulocytes, and also plasma cells. Here, signs of the bone remodeling are also visible. The dark line within the bone, the border of the periosteum before necrosis (red arrow) separates necrotic bone in the center from the new-built bone with a lining of active osteoblasts (blue arrow) on the outside

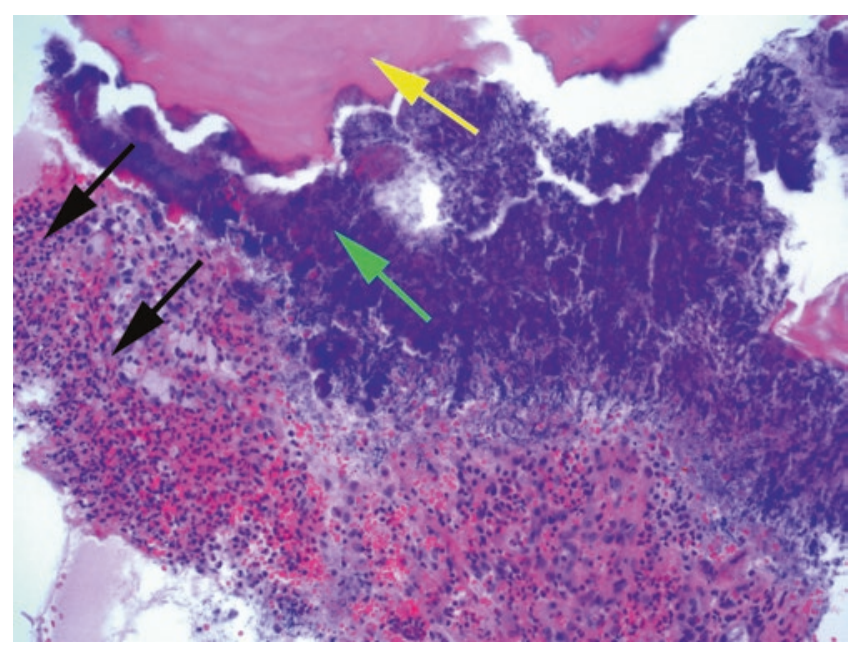

CAssociation of Oral and Maxillofacial Surgeons of India

Fig. 22.6 Actinomyces species (green arrow) frequently can be found next to necrotic bone (yellow arrow) in the jaws. Dense clouds of bacteria affecting the bone leading to deep excavations of the necrotic bone. An inflammatory infiltrate of neutrophil granulocytes and macrophages in fibrin-rich exudate fills the bone marrow (black arrows) as a border which limits bacterial growth parts with empty osteocytic lacunae next to viable osteocytes so that there are necrotic areas of the bone that harbor parts of the viable bone. Inflammatory infiltrates are seen in nearly all cases consisting of granulocytes and lymphocytes. The obliteration of mainly the segmental arteries is not existent in every case [28]. The periosteum is usually viable [5].

\subsubsection{Symptoms}

The classical symptoms are the visible necrotic bone and pain in case of an additional infection of the bone so that the symptoms are very unspecific.

\subsubsection{Complications}

The typical complications are the progress of the initial small necrosis so that big parts of the bone might be affected by the osteonecrosis. Since the bone is no longer covered with soft tissue, an additive infection can cause typical symptoms and leads to a shift to a different stage of the osteonecrosis. Major complications are the loss of the integrity of the bone and extraoral fistulas or abscesses.

The quality of life might essentially get affected in patients with medication-related osteonecrosis of the jaws [29].

\subsubsection{Diagnosis}

The diagnosis is primarily a clinical one if all criteria are fulfilled (see definition). Unfortunately, there is no sensitive radiological tool that shows neither the exact location of the osteonecrosis nor the extent of the necrosis [30]. Once there is a destruction of the bone due to the infection, changes can be seen in the radiologic pictures. But these changes are not specific for the changes (Figs. 22.7 and 22.8a, b). There is evidence that the potential osteonecrosis can be detected at a very early stage via scintigraphs [31].

\subsubsection{Differential Diagnosis}

Regarding the clinical picture, osteomyelitis and osteoradionecrosis are the other typical potential diagnoses. In addition, malignant diseases should be ruled out, especially the ones why the bisphosphonates were given in the first place, e.g., breast cancer or prostate cancer.

\subsubsection{Therapy}

The osteonecroses should be treated since the lack of treatment usually ends in the progression with more extended areas of necrotic bone and a potential switch in the stage of the osteonecrosis.

There are several approaches in the therapy of the bisphosphonate-associated osteonecrosis of the jaws. Smaller osteonecroses can be treated conservatively or with a moderate surgical intervention. Perioperative antibiotic treatment should be initiated. After the debridement or resection of the necrotic bone, a plastic coverage of the bone should be performed. 
Fig. 22.7 This is the same patient as seen in picture 4 There are nearly no changes visible that indicate the existence of osteonecrosis. A very typical change that can be seen is the thickened and sclerotic lamina dura in the mandible and the honeycomb pattern of the bone due to the sclerotic trabeculae of the bone

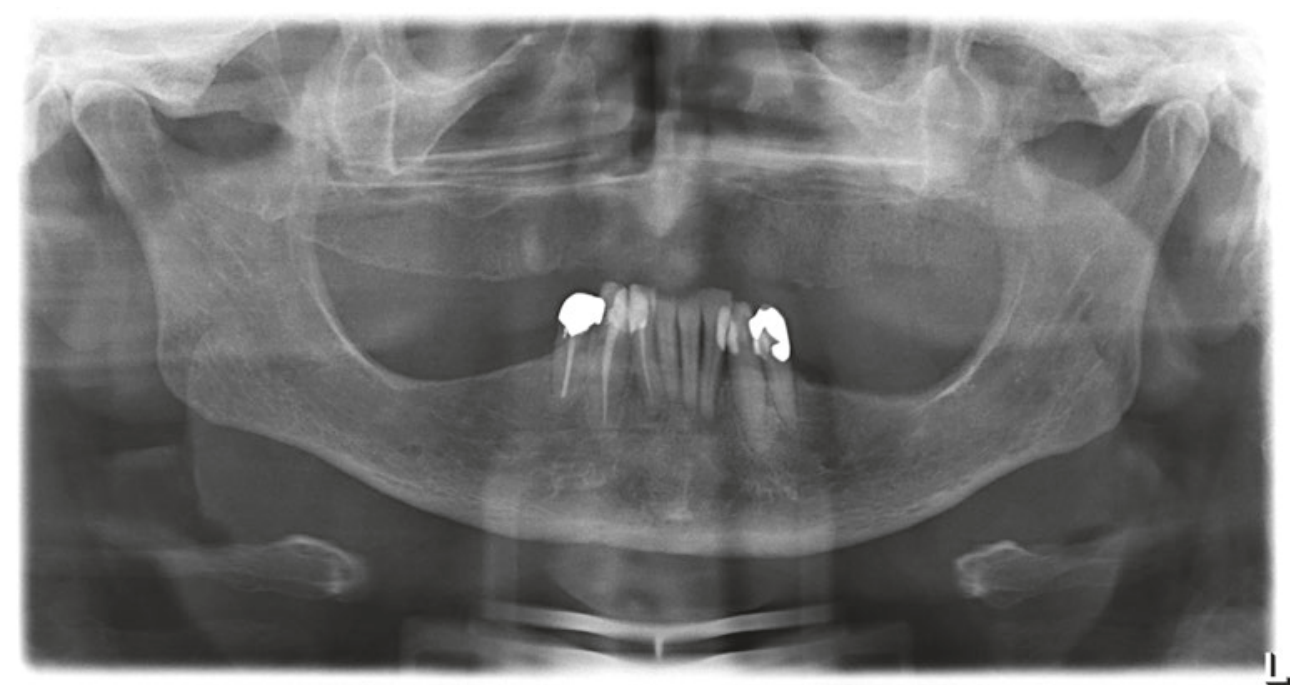

(CAssociation of Oral and Maxillofacial Surgeons of India

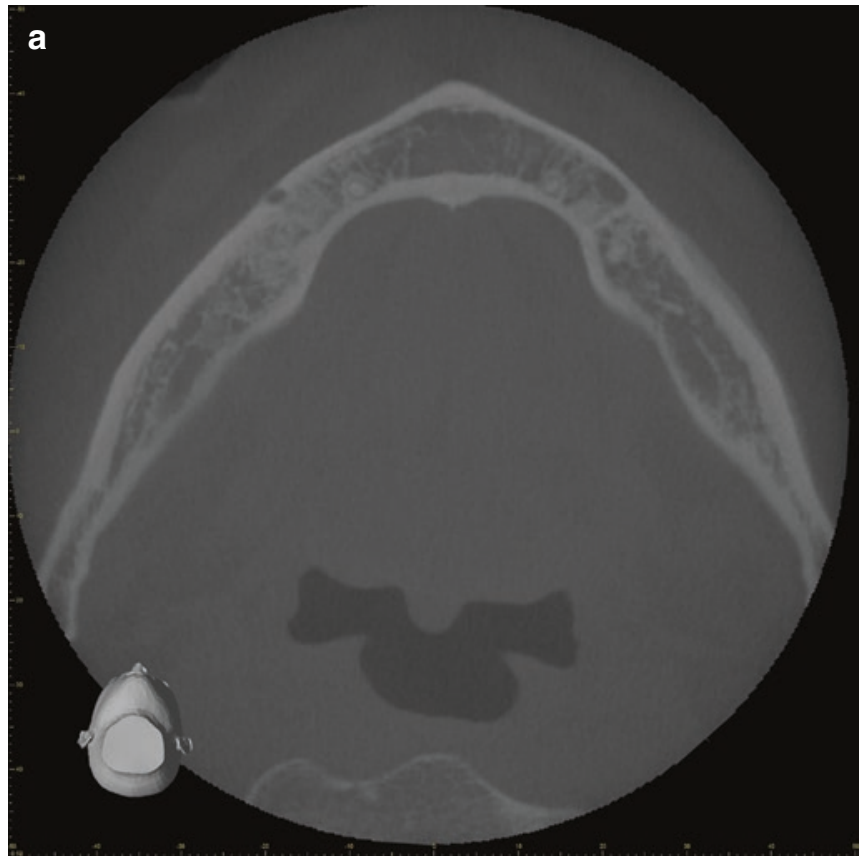

CAssociation of Oral and Maxillofacial Surgeons of India

Fig. 22.8 Two slices from a cone beam CT from a patient with a malignant primary disease and bisphosphonate intake. (a) Axial slice from a patient with osteonecrosis of the left and the right mandible. On the right-hand side, a thickening of the cortical bone can be observed and sclerosis of the cancellous bone. On the left-hand side, the perios-

Extended or multiple osteonecrotic areas should be resected. This might include an inpatient setting with the postoperative use of a nasogastric tube and antibiotic iv treatment [32].

\subsubsection{Prognosis}

The recurrence rate of the osteonecrosis depends on the mode of therapy and is the lowest for surgical interventions

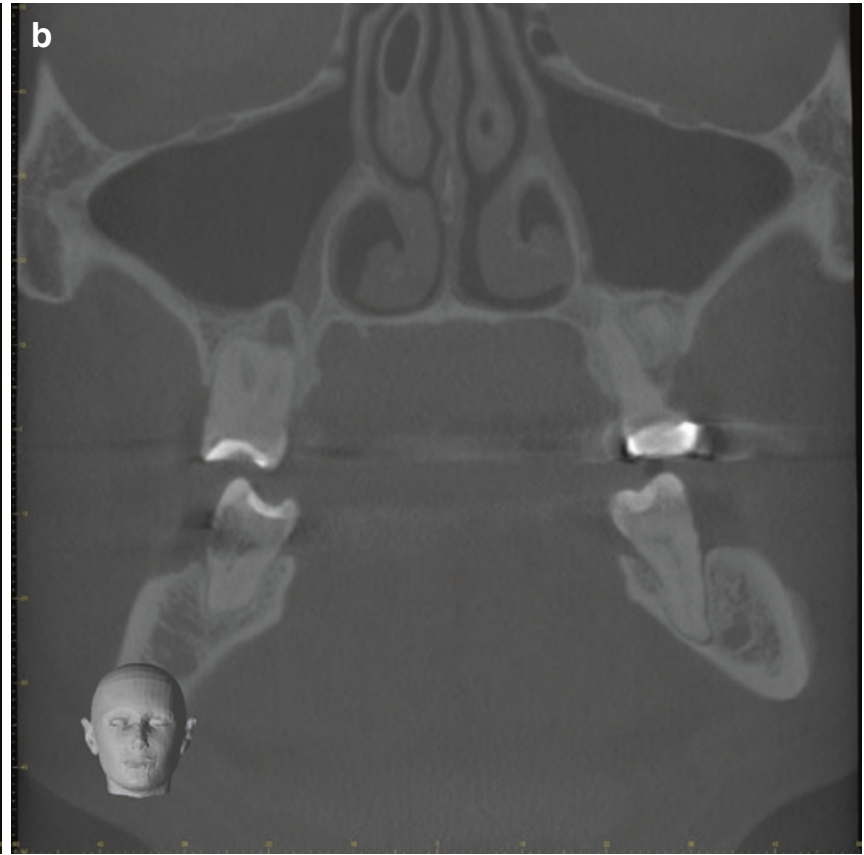

teal reaction is visible. (b) Coronal slice with a periapical translucency of the molar on the right-hand side as a potential trigger of the osteonecrosis that reached the level of the alveolar nerve canal. On the vestibular side of the left mandible the periosteal reaction can be seen

and the highest for purely conservative treatments. In the latter case, complete closure of the mucosal membrane cannot always be achieved [32].

\subsubsection{Prevention}

It should be differed between preventive measures before initiating a bisphosphonate therapy and measures during or 
after the course of bisphosphonate therapy. Since the bisphosphonate-associated osteonecrosis of the jaws is usually triggered by an oral wound, potential dental foci should be eliminated before the start of bisphosphonate therapy [32].

If surgery needs to be performed in patients with bisphosphonate therapy, anti-microbiological prophylaxis should be performed [32].

\subsubsection{Osteonecrosis of the Jaws Due to Other Medications but Bisphosphonates}

There are other medications that can cause osteonecrosis of the jaws. The definition is the same compared to the osteonecrosis due to bisphosphonates.

\subsubsection{Denosumab}

The most frequent osteonecroses can be observed in patients with denosumab that is administered in the same patients that receive bisphosphonates. The definition, etiology, classification, symptoms, complications, diagnoses, differential diagnoses, therapy, prognosis, and prevention are the same or similar to the ones of the bisphosphonate-associated osteonecrosis of the jaws.

The pathogenesis might be different since denosumab only affects the RANK/RANKL/OPG mechanism that is predominant in the communication between osteoblasts and osteoclasts. Therefore, an impact on the soft tissues including the vessels seems unlikely so that the likewise reduced bone remodeling seems to be the major component in the development of the denosumab-associated osteonecrosis of the jaws.

\subsubsection{Sunitinib}

Sunitinib is an inhibitor of a tyrosine kinase and therefore has a less specific mode of action. It is given in patients with gastrointestinal cancers, renal cell carcinoma, and pancreatic neuroendocrine tumors [33]. A side effect in the use of sunitinib is the development of osteonecroses of the jaws [34].

\subsubsection{Imatinib}

Imatinib is another tyrosine kinase inhibitor. Osteonecrosis in imatinib-only use has been described [35].

\subsubsection{Bevazicumab}

Bevazicumab is a vascular endothelial growth factor(VEGF-) inhibitor and is used in several oncologic treatment concepts, e.g., breast cancer, colorectal cancer, and lung cancer. Several cases of osteonecroses of the jaws have been described [36, 37].

\subsubsection{Ziv-aflibercept}

Ziv-aflibercept is a recombinant vascular endothelial growth factor- (VEGF-) receptor. Several cases of the development of osteonecroses of the jaws have been described in patients that did not receive any of the other typical medications causing osteonecrosis [38]. In the pathogenesis, the antiangiogenic factor might be the reason for the development of the osteonecrosis.

\subsubsection{Everolimus}

Everolimus is a mammalian Target of Rapamycin (mTOR) inhibitor. It is used in several oncologic diseases and was associated with the development of osteonecroses in the jaws [39].

\subsubsection{Corticosteroids}

There is evidence that the use of corticosteroids increases the risk of osteonecrosis development of the jaws [40]. The femoral head osteonecrosis has been described in patients with long-term corticosteroid use [41].

It should be stated that the use of several of these agents in combination might further increase the risk of osteonecrosis development.

\subsubsection{Crystal Meth}

Next to therapeutically used agents, some drugs are responsible for the development of osteonecroses. Crystal Meth is the crystalline form of methamphetamine hydrochloride. It functions as a sympathomimetic and has a very high potential for abuse and dependency. One side effect is the meth mouth with serious tooth and damage to the oral cavity including the development of osteonecroses [42]. The exact mechanism of osteonecrosis development is unclear. Methamphetamine causes the release of noradrenaline which increases the blood pressure by the increase of the peripheral blood vessel resistance.

\section{References}

1. Bilezikian JP, Raisz LG, Rodan GA. Principles of bone biology. San Diego: Academic Press; 2002.

2. Bartl R, von Tresckow E, Bartl C. Bisphosphonat-manual. Berlin: Springer; 2006.

3. Liu W, Zhang X. Receptor activator of nuclear factor-kappaB ligand (RANKL)/RANK/osteoprotegerin system in bone and other tissues (review). Mol Med Rep. 2015;11(5):3212-8.

4. Baltensperger MM, Eyrich GK. Osteomyelitis of the jaws. Berlin: Springer; 2009.

5. Marx RE, Tursun R. Suppurative osteomyelitis, bisphosphonate induced osteonecrosis, osteoradionecrosis: a blinded histopathologic comparison and its implications for the mechanism of each disease. Int J Oral Maxillofac Surg. 2012;41(3):283-9. 
6. Muller-Richter UD, Roldan JC, Mortl M, Behr M, Reichert TE, Driemel O. SAPHO syndrome with ankylosis of the temporomandibular joint. Int J Oral Maxillofac Surg. 2009;38(12):1335-41.

7. Marx RE. Osteoradionecrosis: a new concept of its pathophysiology. J Oral Maxillofac Surg. 1983;41(5):283-8.

8. Schuurhuis JM, Stokman MA, Witjes MJ, Dijkstra PU, Vissink A, Spijkervet FK. Evidence supporting pre-radiation elimination of oral foci of infection in head and neck cancer patients to prevent oral sequelae. A systematic review. Oral Oncol. 2015;51(3):212-20.

9. Walter C, Sagheb K, Bitzer J, Rahimi-Nedjat R, Taylor KJ. Analysis of reasons for osteonecrosis of the jaws. Clin Oral Investig. 2014;18(9):2221-6.

10. Krüger M, Hautmann M, Bartella A, Al-Nawas B, Grötz KA. S2kLeitlinie: Infizierte Osteoradionekrose (IORN) der Kiefer. AWMF Online 2018.

11. Annane D, Depondt J, Aubert P, Villart M, Gehanno P, Gajdos P, et al. Hyperbaric oxygen therapy for radionecrosis of the jaw: a randomized, placebo-controlled, double-blind trial from the ORN96 study group. J Clin Oncol. 2004;22(24):4893-900.

12. Sultan A, Hanna GJ, Margalit DN, Chau N, Goguen LA, Marty FM, et al. The use of hyperbaric oxygen for the prevention and Management of Osteoradionecrosis of the jaw: a Dana-Farber/ Brigham and Women's Cancer center multidisciplinary guideline. Oncologist. 2017;22(3):343-50.

13. Pitak-Arnnop P, Sader R, Dhanuthai K, Masaratana P, Bertolus C, Chaine A, et al. Management of osteoradionecrosis of the jaws: an analysis of evidence. Eur J Surg Oncol. 2008;34(10):1123-34.

14. Hagelauer N, Ziebart T, Pabst AM, Walter C. Bisphosphonates inhibit cell functions of HUVECs, fibroblasts and osteogenic cells via inhibition of protein geranylgeranylation. Clin Oral Investig. 2015;19(5):1079-91.

15. Marx RE. Pamidronate (Aredia) and zoledronate (Zometa) induced avascular necrosis of the jaws: a growing epidemic. J Oral Maxillofac Surg. 2003;61(9):1115-7.

16. Ruggiero SL, Dodson TB, Fantasia J, Goodday R, Aghaloo T, Mehrotra B, et al. American association of oral and maxillofacial surgeons position paper on medication-related osteonecrosis of the jaw--2014 update. J Oral Maxillofac Surg. 2014;72(10):1938-56.

17. Hansen T, Kirkpatrick CJ, Walter C, Kunkel M. Increased numbers of osteoclasts expressing cysteine proteinase cathepsin $\mathrm{K}$ in patients with infected osteoradionecrosis and bisphosphonateassociated osteonecrosis--a paradoxical observation? Virchows Arch. 2006;449(4):448-54.

18. Rugani P, Walter C, Kirnbauer B, Acham S, Begus-Nahrman Y, Jakse N. Prevalence of medication-related osteonecrosis of the jaw in patients with breast cancer, prostate cancer, and multiple myeloma. Dent J (Basel). 2016;4(4):32.

19. Walter C, Al-Nawas B, Frickhofen N, Gamm H, Beck J, Reinsch L, et al. Prevalence of bisphosphonate associated osteonecrosis of the jaws in multiple myeloma patients. Head Face Med. 2010;6:11.

20. Walter C, Al-Nawas B, Grotz KA, Thomas C, Thuroff JW, Zinser $\mathrm{V}$, et al. Prevalence and risk factors of bisphosphonateassociated osteonecrosis of the jaw in prostate cancer patients with advanced disease treated with zoledronate. Eur Urol. 2008;54(5):1066-72.

21. Grötz KA, Piesold U, Al-Nawas B. Bisphosphonate-assoziierte Osteonekrose (BP-ONJ) und andere Medikamenten-assoziierte Kiefernekrosen. AWMF [Internet]. 2012; (007/091):1-18.

22. Walter C, Grotz KA, Kunkel M, Al-Nawas B. Prevalence of bisphosphonate associated osteonecrosis of the jaw within the field of osteonecrosis. Support Care Cancer. 2007;15(2):197-202.
23. Koch FP, Merkel C, Ziebart T, Smeets R, Walter C, Al-Nawas B. Influence of bisphosphonates on the osteoblast RANKL and OPG gene expression in vitro. Clin Oral Investig. 2012;16(1):79-86.

24. Walter C, Klein MO, Pabst A, Al-Nawas B, Duschner H, Ziebart T. Influence of bisphosphonates on endothelial cells, fibroblasts, and osteogenic cells. Clin Oral Investig. 2010;14(1):35-41.

25. Ziebart T, Pabst A, Klein MO, Kammerer P, Gauss L, Brullmann $\mathrm{D}$, et al. Bisphosphonates: restrictions for vasculogenesis and angiogenesis: inhibition of cell function of endothelial progenitor cells and mature endothelial cells in vitro. Clin Oral Investig. 2011;15(1):105-11.

26. Pabst AM, Ziebart T, Ackermann M, Konerding MA, Walter C. Bisphosphonates' antiangiogenic potency in the development of bisphosphonate-associated osteonecrosis of the jaws: influence on microvessel sprouting in an in vivo 3D Matrigel assay. Clin Oral Investig. 2014;18(3):1015-22.

27. Saia G, Blandamura S, Bettini G, Tronchet A, Totola A, Bedogni $\mathrm{G}$, et al. Occurrence of bisphosphonate-related osteonecrosis of the jaw after surgical tooth extraction. J Oral Maxillofac Surg. 2010;68(4):797-804.

28. Hansen T, Kunkel M, Springer E, Walter C, Weber A, Siegel E, et al. Actinomycosis of the jaws--histopathological study of 45 patients shows significant involvement in bisphosphonateassociated osteonecrosis and infected osteoradionecrosis. Virchows Arch. 2007;451(6):1009-17.

29. Kyrgidis A, Triaridis S, Kontos K, Patrikidou A, Andreadis C, Constantinidis $\mathrm{J}$, et al. Quality of life in breast cancer patients with bisphosphonate-related osteonecrosis of the jaws and patients with head and neck cancer: a comparative study using the EORTC QLQ-C30 and QLQ-HN35 questionnaires. Anticancer Res. 2012;32(8):3527-34.

30. Berg BI, Mueller AA, Augello M, Berg S, Jaquiery C. Imaging in patients with bisphosphonate-associated osteonecrosis of the jaws (MRONJ). Dent J (Basel). 2016;4(3):E29.

31. Thomas C, Spanidis M, Engel C, Roos FC, Frees S, Neisius A, et al. Bone scintigraphy predicts bisphosphonate-induced osteonecrosis of the jaw (BRONJ) in patients with metastatic castration-resistant prostate cancer (mCRPC). Clin Oral Investig. 2016;20(4):753-8.

32. Otto S, Pautke C, Van den Wyngaert T, Niepel D, Schiodt M. Medication-related osteonecrosis of the jaw: prevention, diagnosis and management in patients with cancer and bone metastases. Cancer Treat Rev. 2018;69:177-87.

33. Imbulgoda A, Heng DY, Kollmannsberger C. Sunitinib in the treatment of advanced solid tumors. Recent Results Cancer Res. 2014;201:165-84.

34. Koch FP, Walter C, Hansen T, Jager E, Wagner W. Osteonecrosis of the jaw related to sunitinib. Oral Maxillofac Surg. 2011;15(1):63-6.

35. Viviano M, Rossi M, Cocca S. A rare case of osteonecrosis of the jaw related to imatinib. J Korean Assoc Oral Maxillofac Surg. 2017;43(2):120-4.

36. Estilo CL, Fornier M, Farooki A, Carlson D, Bohle G 3rd, Huryn JM. Osteonecrosis of the jaw related to bevacizumab. J Clin Oncol. 2008;26(24):4037-8.

37. Santos-Silva AR, Belizario Rosa GA, Castro Junior G, Dias RB, Prado Ribeiro AC, Brandao TB. Osteonecrosis of the mandible associated with bevacizumab therapy. Oral Surg Oral Med Oral Pathol Oral Radiol. 2013;115(6):e32-6.

38. Mawardi H, Enzinger P, McCleary N, Manon R, Villa A, Treister $\mathrm{N}$, et al. Osteonecrosis of the jaw associated with ziv-aflibercept. J Gastrointest Oncol. 2016;7(6):E81-E7.

39. Yamamoto D, Tsubota Y, Utsunomiya T, Sueoka N, Ueda A, Endo $\mathrm{K}$, et al. Osteonecrosis of the jaw associated with everolimus: a case report. Mol Clin Oncol. 2017;6(2):255-7. 
40. Saad F, Brown JE, Van Poznak C, Ibrahim T, Stemmer SM, Stopeck AT, et al. Incidence, risk factors, and outcomes of osteonecrosis of the jaw: integrated analysis from three blinded active-controlled phase III trials in cancer patients with bone metastases. Ann Oncol. 2012;23(5):1341-7.

41. Drescher W, Bunger MH, Weigert K, Bunger C, Hansen ES. Methylprednisolone enhances contraction of porcine femoral head epiphyseal arteries. Clin Orthop Relat Res. 2004;423:112-7.
42. Pabst A, Castillo-Duque JC, Mayer A, Klinghuber M, Werkmeister R. Meth mouth-a growing epidemic in dentistry? Dent J (Basel). 2017;5(4):E29.

Open Access This chapter is licensed under the terms of the Creative Commons Attribution 4.0 International License (http://creativecommons. org/licenses/by/4.0/), which permits use, sharing, adaptation, distribution and reproduction in any medium or format, as long as you give appropriate credit to the original author(s) and the source, provide a link to the Creative Commons license and indicate if changes were made.

The images or other third party material in this chapter are included in the chapter's Creative Commons license, unless indicated otherwise in a credit line to the material. If material is not included in the chapter's Creative Commons license and your intended use is not permitted by statutory regulation or exceeds the permitted use, you will need to obtain permission directly from the copyright holder. 